\title{
Benign Multicystic Peritoneal Mesothelioma: Case Report
}

Agnaldo Viana Pereira Neto ${ }^{1}$, Nathanael de Freitas Pinheiro Júnior ${ }^{2}$, Renata Campos Simões Cabral ${ }^{1,2}$, Isabelle Torres ${ }^{2}$, Hilton Pina ${ }^{1}$, Antônio Carlos Travessa ${ }^{1}$ and Milena Bastos Brito ${ }^{3^{*}}$

${ }^{1}$ Department of Obstetrics and Gynecology, Federal University of Bahia, Salvador-BA, Brazil

${ }^{2}$ Departament of Pathology and Forensic Medicine, Federal University of Bahia, Salvador -BA, Brazil

${ }^{3}$ Department of Obstetrics and Gynecology, Bahiana School of Medicine and Public Health, Salvador-BA, Brazil

*Corresponding author: Milena Bastos Brito, Department of Obstetrics and Gynecology, Bahiana School of Medicine and Public Health, Av. D. João VI, 275-Brotas, Salvador-BA 40285 001, Brazil, Tel: +55(71) 3276-8265; Fax: +55(71) 3276-8202; E-mail: milenabrito@bahiana.edu.br

Rec date: April 7, 2016; Acc date: June 13, 2016; Pub date: June 19, 2016

Copyright: $\odot 2016$ Neto AVP, et al. This is an open-access article distributed under the terms of the Creative Commons Attribution License, which permits unrestricted use, distribution, and reproduction in any medium, provided the original author and source are credited.

\begin{abstract}
Benign multicystic peritoneal mesothelioma (BMPM) is a rare tumor of slow growth, with low malignant potential and which is part of the differential diagnosis of pelvic cysts. We described a case report: 55 years old woman, asymptomatic, with ultrasonographic findings of multicystic pelvic image, submitted to exploratory laparotomy and, at the inventory of the cavity, multiple cysts were present, adhered to the omentum. Omentectomy and resection of the cysts was performed followed by complete hysterectomy and bilateral oophorectomy and salpingectomy. Macroscopic examination of the surgical specimen revealed a cystic lesion with translucent content and histological features compatible with BMPM. In the immediate postoperative period, the patient developed vasomotor symptoms secondary to hypoestrogenism, which ceased spontaneously. She underwent a new surgical approach for recurrence after two years. Since the pathogenesis and etiology of BMPM are not well defined, it is very important to describe clinical practices, to better understand the diagnosis, prognosis and management of this disease.
\end{abstract}

Keywords: Mesothelioma cystic; Diagnosis; Adnexal diseases; Peritoneal neoplasm

\section{Introduction}

Benign Multicystic Peritoneal Mesothelioma (BMPM) is a rare disease. Today approximately 140 cases have been reported all over the world. It occurs particularly among women during reproductive years.

Common symptoms are chronic pain in lower abdomen and a pelvic mass. Many cases reported previous pelvic surgery, endometriosis and pelvic inflammatory disease [1]. The pathogenesis of BMPM is unknown. Some authors believe that it is an inflammatory process, while others neoplastic lesion [2,3]. Treatment includes surgical approach with extensive lesions resection [4]. However other less aggressive therapies have been advocated for younger women [5]. It is important to follow-up these patients after surgical treatment because of the possibility of recurrences and tendency to transform into malignancy [5] We will report a case of BMPM followed in our service, then we will have a discussion and review of the literature about this rare disease.

\section{Case Report}

An informed consent free and clear was provided by the patient as described by the Research Ethics Committee, for this publication. Woman 55 years, asymptomatic, with incidental finding of pelvic multicystic measuring $9.1 \times 7.6 \times 7.8 \mathrm{~cm}$ to routine transvaginal ultrasound, on April 20th, 2011, which showed anteverted uterus with volume of $154 \mathrm{~cm}^{3}$ with the presence of fibroid nodules, the largest one measuring $3.3 \times 2.8 \mathrm{~cm}$. Endometrium measuring $18.0 \mathrm{~mm}$. Ovaries not displayed. Antecedents, the patient reported five previous pregnancies with a history of four repeat abortions, followed by uterine curettage.
The last pregnancy was 18 years ago. She denied any history of pelvic surgery or sexually transmitted diseases. On physical examination, palpable a mass in the left iliac fossa with difficult delimitation by thick adipose tissue.

The gynecological examination showed no changes. CEA level 0.70 $\mathrm{mg} / \mathrm{mL}$. An magnetic resonance of the pelvis was requested on May 27th, 2011, which showed the presence of moderate/severe amount of fluid in the abdominal cavity, loculated, as well as nodular areas with high signal on T1 and T2, including the sequences with fat saturation, suggesting hematic content, questioned carcinomatosis.

Uterus in AVF, enlarged, lobulated, myometrium heterogeneous with multiple nodular lesions intramural, subserosa and submucosal, the biggest of them with $7.0 \mathrm{~cm}$ in fundus, suggesting leyomiomas. Ovaries and iliac vessels without changes. Laparotomy revealed numerous cysts adhered to the omentum (Figure 1).

Omentectomy and resection of the cysts was performed followed by complete hysterectomy and bilateral oophorectomy and salpingectomy. Macroscopically observed uterus with leiomyomas and ovaries without changes. Peritoneal cysts weighing $450 \mathrm{~g}$, represented by numerous cysts of varying sizes, smooth surfaces, translucent, grouped in clusters. Microscopic examination revealed cavities of cysts coated single row of flat cells with scant cytoplasm varying the eosinophilic (Figure 2). Some cells have cilia. No nuclear or mitotic hyperchromasia. The cysts were separated by loose stroma. Immunohistochemically study revealed positivity of cells lining the cysts for calretinin and pan-citoceratona (AE1/AE3).

The cells were negative for CD34 (Figures 3A and 3B). Two months after surgery, the patient reported vasomotor symptoms (palpitations, sweating and hot flushes) and high FSH levels, which stopped without medications. 


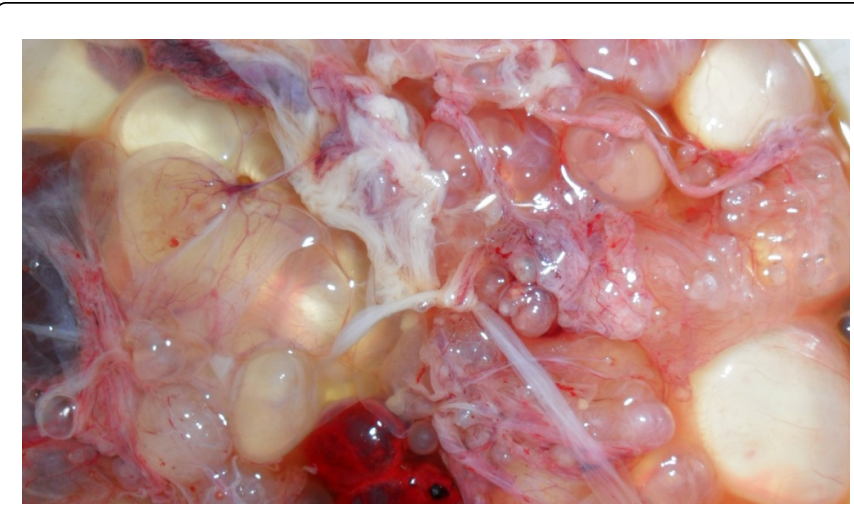

Figure 1: Multiple peritoneal cysts of varying size with thin walls lined by flattened mesothelial cells, grouped as clusters.

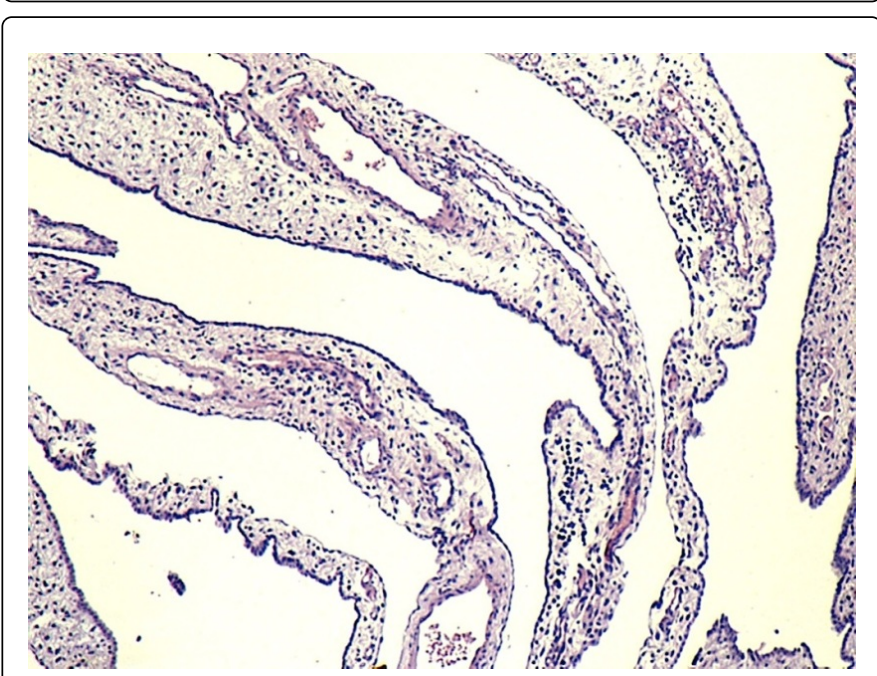

Figure 2: Microscopy showed cystic cavities lined by cuboidal epithelium or simple flat, separated by vascularized edematous stroma.

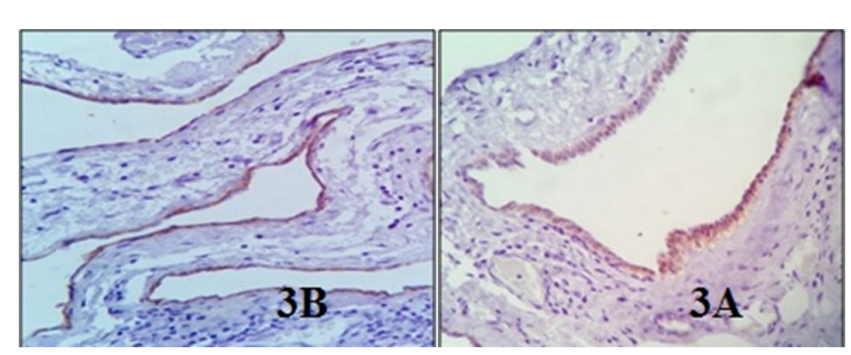

Figure 3: 3A (right): Mesothelial cells without atypia, positive for calretinin. 3B (left): Mesothelial cells positive for AE1/AE3.

The patient remained in attendance for a year and eight months asymptomatic. Postoperatively held in August 2012 CEA: $0.81 \mathrm{ng} / \mathrm{mL}$, CA 125: $6.60 \mathrm{U} / \mathrm{mL}$. In October 2012 abdominal ultrasound showed cystic mass in supraumbilical region, mild hepatic steatosis and cholelithiasis. After this period, she presented bulges in the abdominal wall above and infraumbilical, and pain in the right hypochondrium.
Computerized tomography of the abdomen showed incisional hernias, gallstones, and circumscribed and net free fluid in the peritoneal cavity, unspecific for only residual ascites or peritoneal dissemination of representing blastomatosis process.

The patient underwent a new exploratory laparotomy on November, 2013, with cholecystectomy, hernia repair and excision of peritoneal cystic lesions whose microscopic analysis revealed cuboidal or flattened cells without atypia, confirming the suspicion of recurrence of mesothelial cysts.

\section{Discussion}

Benign multicystic peritoneal mesothelioma (BMPM) is also known as benign cystic mesothelioma peritoneum or multilocular cysts inclusion peritoneal [6]. It is a rare disease that occurs most often in women of reproductive age and is associated with a history of prior abdominal surgery, endometriosis or inflammatory pelvic disease [6]. There are even cases reports in men [7] (ratio of 5 women:1 man) [8] and children [9].

The BMPM stems from peritoneal mesothelium which mainly involves the peritoneum and uterus, fallopian tubes, ovaries, rectum, cervix and the area of Douglas fornix, with rare involvement of the upper abdomen and retroperitoneal. Some cases have also been described in vaginal tunic [5]. Most papers about the disease are case reports [1,5,8] and clinical series, the largest with 17 patients [8].

The MBM is characterized by solitary or multiple cysts, with fine septa, multiloculated and filled with serous fluid or blood. The size comprises lesions a few millimeters to 30 centímetros [10]. The microscopy shows up multiple cystic spaces lined by cuboidal epithelium. There is no atypia or mitosis. Cysts are separated by fibrous septa with areas of chronic inflammation and proliferation stromal cells [11].

As identified in this case, the pathological diagnosis of BMPM includes the presence of peritoneal cysts grouped as "grape clusters", filled with citrus serous or hemorrhagic and microscopically coated with flat or cuboidal epithelium [12].

There are two types of mesotheliomas benign: the BMPM discussed in this case and the mesothelioma papillary well differentiated. They exhibit frequent recurrence, as noted in the patient described, which occurred two years after the first surgery [12]. The other mesotheliomas are defined as malignancies.

Differential diagnoses include cystic epithelial tumors of the ovary, endometriosis, cystic lymphangioma and cistic adenomatoid tumor [13]. The lymphangioma is restricted to the mesentery, omentum, mesocolon and rarely affects the ovaries. The microscopy note lymphoid tissue and smooth muscle. Adenomatoid cysts, by the way, are more difficult to differentiate because the macroscopic and the histology are similar, but cysts are usually accompanied by a solid component. There are reports of BMPM associated with tumor adenomatoide [10].

The BMPM is commonly asymptomatic, as the case described, and often an incidental finding on physical, imaging or intraoperative and can affect organs by contiguity [10]. By achieving large size, can cause abdominal pain, painful palpable mass or may only cause bloating. Other symptoms include ascites, weight loss, nausea, vomiting and constipation. 
Ultrasonography reveals a multicystic mass, richly vascularized and without calcification. CT scan typically shows a multicystic lesion with density similar to blood. Magnetic resonance, however, shows a multilocular cystic lesion with low intensity in $\mathrm{T} 1$ and mean intensity in T2 [5].

The definitive diagnosis is made by histopathologic study [5]. The electron microscopy or immunohistochemically can assist diagnosis. Serum tumor markers such as CA-125, CEA, CA-15.3, CA-19.9, ferritin, 2-microglobulin are usually normals [14-16] with one case reported with elevated CA-19.9 and regressed after the cirurgic treatment [15].

The immunohistochemically study is important to exclude possible mimic neoplasms cystic. Generally positive markers include calretinin, D2-40, CK5/6 and WT-1, mesodermal markers present in epithelia, especially the mesothelium. Markers MOC-31, PAX8, BG8, Ber-EP4, B72.3, CEA, and CDX-2 are frequently negatives [17,18]. Most often used an epithelial marker (cytokeratin), and CD34, an endothelial marker that can also be used to exclude lymphangioma or other suspected vascular neoplasms. In this study the patient was positive for calretinin and cytokeratin, and negative for $\mathrm{CD} 34$, reinforcing the mesothelial origin in the epithelium injury.

The etiology still controverse, but there are some proposed hypotheses to clarify it. The inflammatory hypothesis postulates that tumors arise from a peritoneal inflammation caused by chronic peritoneal irritation, proliferation, and cystic formation of mesothelial cells. Corroborate to this hypothesis association with previous abdominal surgery, endometriose $[5,14,19]$ and mediterranean fever familiar $[20,10]$. Neoplastic origin was proposed before the slow growth characteristics, but progressive untreated injuries and also a marked tendency to recur after resection [21]. But the histological features are typical of a benign lesion. The genetic hypothesis arose from the description of family members of mesothelioma cases in association with other conditions such as cataracts and diverticulitis. Finally, abnormalities in the development could lead to formation of cysts in the mesothelial $[5,10]$. This injury has no association with exposure to amianto [8].

Although BMPM is associated with a favorable prognosis in a short time, because it is a completely benign condition; in long time it has been described about $50 \%$ of postoperative recidive [13]. There is evidence that malignant transformation could occur. There are two cases described of malign processing [22,23].

The clinical importance of the differential diagnosis of cystic tumors of the ovaries is the therapeutic choice. Once set define like a BMPM the treatment is local excision, preserving the ovaries [10].

Surgical treatment is the most effective. It is recommended complete removal of cysts and also omentectomy. For younger patients there are more conservative treatments proposals, such as sclerosing therapy with tetracycline, continuous peritoneal hyperthermic perfusion with cisplatin and doxorubicin, anti-estrogenic drugs [12]. It is known, however, that none of these conservative treatments have success rates as surgery. Adjuvant chemotherapy and radiation have little value because of benign character of BMPM. The use of Tamoxifen has been suggested as mesothelioma express estrogen and progesterone receptors, but the effect of estrogen is not fully elucidate [12].

A long follow-up period is always necessary [5], although there is still no consensus on when and what additional tests should be ordered.
The description of this case is important to the scientific literature because BMPM is a very rare pathology and the differential diagnostic with pelvic cysts. It's important have a careful long-term follow-up, because high risk of recidive despite benign characteristics.

\section{References}

1. McCaffrey JC, Foo FJ, Dalal N, Siddiqui K (2009) Benign multicystic peritoneal mesothelioma associated with hydronephrosis and colovesical fistula formation: report of a case. Tumori 95: 808-810.

2. Tentes AA, Zorbas G, Pallas N, Fiska A (2012) Multicystic peritoneal mesothelioma. Am J Case Rep 13: 262-264.

3. Yang DM, Jung DH, Kin H, Kang JH, Kim SH, et al. (2004) Retroperitoneal cystic masses: CT, clinical and pathologic findings and literature review. Radiographics 24: 1353-1365.

4. Sethna K, Mohamed F, Marchettini P, Elias D, Sugarbaker PH (2003) Peritoneal cystic mesothelioma: a case series. Tumori 89: 31-35.

5. Cavallaro A, Berretta M, Lo Menzo E, Cavallaro V, Zanghì A, et al. (2011) Cystic peritoneal mesothelioma: report of a case. Surg Today 41: 141-146.

6. Dzieniecka M, Kaluzynski A (2011) Benign multicystic peritoneal mesothelioma (BMPM) - case report and review of the literature. Pol J Pathol 62: 122-124.

7. Sienkowski IK, Russell AJ, Dilly SA, Djazaeri B (1986) Peritoneal cystic mesothelioma: an electron microscopic and immunohistochemical study of two male patients. J Clin Pathol 39: 440-445.

8. Akbayir O, Gedikbasi A, Akyol A, Numanoglu C, Koroglu N, et al. (2011) Benign cystic mesothelioma: a case series with one case complicated by pregnancy. J Obstet Gynaecol Res 37: 1126-1131.

9. Mennemeyer R, Smith M (1979) Multicystic peritoneal mesothelioma: a report with electron microscopy of a case mimicking intra-abdominal cystic hygroma (lymphangioma). Cancer 44: 692-698.

10. Safioleas MC, Constantinos K, Michael S, Konstantinos G, Constantinos $S$, et al. (2006) Benign multicystic peritoneal mesothelioma: a case report and review of the literature. World J Gastroenterol 12: 5739-5742.

11. Scattone A, Pennella A, Giardina C, Marinaccio M, Ricco R, et al. (2001) Polycystic mesothelioma of the peritoneum: Description of four cases. Pathologica 93: 549-555.

12. Kindler HL (2013) Peritoneal mesothelioma: the site of origin matters. Am Soc Clin Oncol Educ Book.

13. Park JY, Kim KW, Kwon HJ, Park MS, Kwon GY, et al. (2008) Peritoneal mesotheliomas: clinicopathologic features, CT findings and differential diagnosis. AJR Am J Roentgenol 191: 814-825.

14. Takemoto S, Kawano R, Honda K, Nakazono A, Shimamatsu K (2012) Benign multicystic peritoneal mesothelioma mimicking recurrence of an ovarian borderline tumor: a case report. J Med Case Rep 6: 126.

15. Pinto V, Rossi AC, Fiore MG, D'Addario V, Cicinelli E (2010) Laparoscopic diagnosis and treatment of pelvic benign multicystic mesothelioma associated with high CA 19.9 serum concentration. J Minim Invasive Gynecol 17: 252-254.

16. Sawh RN, Malpica A, Deavers MT, Liu J, Silva EG (2003) Benign cystic mesothelioma of the peritoneum: a clinicopathologic study of 17 cases and immunohistochemical analysis of strogen and progesterone receptor status. Hum Pathol 34: 369-374.

17. Husain AN, Colby T, Ordonez N, Krausz T, Attanoos R, et al. (2012) Guidelines for pathologic diagnosis of malignant mesothelioma: 2012 update of the consensus statement from the International Mesothelioma Interest Group. Arch Pathol Lab Med 137: 647-667.

18. Doglioni C, Dei Tos AP, Laurino L, Iuzzolino P, Chiarelli C, et al. (1996) Calretinin: a novel immunocytochemical marker for mesothelioma. Am J Surg Pathol 20: 1037-1046.

19. Shakya VC, Agrawal CS, Karki S, Sah PL, Poudel P, et al. (2011) Benign cystic mesothelioma of the peritoneum in a child - case report and review of the literature. Journal of Pediatric Surgery 46: 23-26. 
Citation: Neto AVP, Pinheiro Júnior NFde,Cabral RCS, Torres I, Pina H, et al. (2016) Benign Multicystic Peritoneal Mesothelioma: Case Report . Gen Med (Los Angeles) 4: 253. doi:10.4172/2327-5146.1000253

Page 4 of 4

20. Cuartas JE, Maheshwari A, Qadir R, Cooper AJ, Robinson PG, et al. (2008) Benign multicystic peritoneal mesothelioma in a cesarean-section scar presenting as a fungating mass. Int J Clin Oncol 13: 275-278.

21. González-Moreno S, Yan H, Alcorn KW, Sugarbaker PH (2002) Malignant transformation of "benign" cystic mesothelioma of the peritoneum. J Surg Oncol 79: 243-251.
22. Suhag V, Kaushal V, Sunita BS, Joshi A (2004) Cystic mesothelioma: role of tamoxifen in preventing recurrence in the post-operative setting. Pakistan J Med Res 43.

23. Navarra G, Santin M, Carcoforo P, Sortini A (1996) Peritoneal cystic mesothelioma treated with minimally invasive approach. Surge Endose 10: 60-61. 